\title{
FYI: This Message Will Interrupt You - Texting Impact on Clinical Learning Environment
}

\author{
Irsk Anderson, MD*; Vineet M. Arora, MD, MAPP
}

Section of General Internal Medicine, Department of Medicine, University of Chicago, Chicago, Illinois.

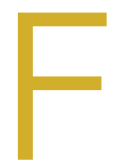

ifteen years ago, beepers with 5-digit call-back numbers were the norm. Pushing a call light button outside the patient's room to flag the desk clerk that a new order had been hand-written was all part of the lived experience of residency. Using that as our baseline, we have clearly come a long way in the way that we communicate with other clinicians in hospitals. Communication among the patient care team in the digital age predominantly involves bidirectional messaging using mobile devices. The approach is both immediate and convenient. Mobile devices can improve work efficiency, patient safety, and quality of care, but their main advantage may be real-time bedside decision support. ${ }^{1,2}$ However, the widespread use of mobile devices for communication in healthcare is not without its concerns. First and foremost, there has been abundant literature around short message service (SMS) use in the healthcare setting, and there are concerns surrounding both threats to privacy and the prevalence and impact of interruptions in clinical care.

The first SMS was sent in 1992. ${ }^{3}$ Text messaging since then has become ubiquitous, even in healthcare, raising concerns around the protection of patient health information under the Health Insurance Portability and Accountability Act (HIPAA). Interestingly, the United States Department of Health and Human Services Office for Civil Rights, enforcer of HIPAA, is tech neutral on the subject. ${ }^{3}$ Multiple studies have assessed physician stances on SMS communication in the healthcare setting using routine, non-HIPAA-compliant mobile phones. Overall, $60 \%-80 \%$ of respondents admitted to using SMS in patient care, while in another study, $72 \%$ and $80 \%$ of Internal Medicine residents surveyed found SMS to be the most efficient form of communication and overall preferred method of communication, respectively., $\mathrm{In}$ terestingly, $82.5 \%$ of those same residents preferred the hospital-based alphanumeric paging system for security purposes, even though Freundlich et al. make a compelling argument that unidirectional alphanumeric paging systems are most certainly less HIPAA compliant, lacking encryption and password protection. ${ }^{5}$ Newer platforms that enable HIPAA-compliant messaging are promising, although they may not be fully adopted by clinical teams without full-scale implementation in hospitals. ${ }^{b}$

\footnotetext{
*Address for correspondence: Irsk Anderson, MD, 5841 S. Maryland Ave, MC 3051, Chicago, IL 60637; Telephone: 773-702-6840; Fax: 773-834-3945; E-mail: ianderso@medicine.bsd.uchicago.edu
}

Received: May 25, 2018; Accepted: June 6, 2018

๑) 2018 Society of Hospital Medicine DOI 10.12788/jhm.3067
In addition to privacy concerns with SMS applications on mobile phones, interruptions in healthcare - be it from phone calls, emails, text messages, or in-person conversations - are common. In fact, famed communication researcher Enrico Coeira has notoriously described healthcare communication as "interrupt-driven." terruptions in the healthcare setting can lead to medication prescription errors, errors in computerized physician order entry, and even surgical procedural errors. . $^{8-10}$

While studies have focused on interruptions in clinical care in the healthcare setting, little is known about how education may be compromised by interruptions due to mobile devices. Text messaging during dedicated conference time can lead to inadequate learning and a sense of frustration among residents. In this issue of the Journal of Hospital Medicine, Mendel et al. performed a quality improvement study involving eight academic inpatient clinical training units with the aim of reducing nonurgent text messages during education rounds. ${ }^{11}$ Their unique interventions included learning sessions, posters, adding alerts to the digital communication platform, and alternative messaging options. Of four sequential interventions, a message alerting the sender that they will be interrupting educational rounds and suggesting a "delayed send" or "send as an FYI" showed the greatest impact, reducing the number of text interruptions per team per educational hour from 0.81 to 0.59 (95\% Cl 0.51-0.67). When comparing a four-week pre-intervention sample with a four-week end-intervention sample, the percentage of nonurgent messages decreased from $82 \%$ to $68 \%(P<.01)$.

While these results are promising, challenges to large-scale implementation of such a program exist. Buy-in from the ancillary healthcare team is critical for such interventions to succeed and be sustained. It also places a burden of "point triage" on the healthcare team members, who must assess the patient situation and determine the level of urgency and whether to immediately interrupt, delay interrupt or send an FYI message. For example, in the study by Mendel et al., ${ }^{11}$ it is noteworthy that urgent patient care issues were mislabeled as "FYI" in $2 \%$ of patients. While this is a seemingly low rate, even one of these mislabeled messages could result in significant adverse patient outcomes and should be considered a "never event." Finally, the study used a messaging platform with programming flexibility and IT personnel to assist. This could be cost prohibitive for some programs, especially if rolled out to an entire institution.

Communication is critical for effective patient care and unfortunately, the timing of such communication is often not 
orderly but rather, chaotic. Text message communication can introduce interruptions into all aspects of patient care and education, not only dedicated learning conferences. If the goal is for all residents to attend all conferences, it seems impossible (and likely dangerous) to eliminate all messaging interruptions during conference hours. Nevertheless, it is worth noting that Mandel et al. have moved us creatively toward that goal with a multifaceted approach. ${ }^{11}$ Future work should address more downstream outcomes, such as objective resident learning retention and adverse patient events relative to the number of interruptions per educational hour. If such studies showed improved learning outcomes and fewer adverse patient events, the next step would be to further strengthen and refine their protocol with real-time and scheduled feedback sessions between providers and other patient care team members in addition to the continued search for additional innovative approaches. In addition, combining artificial intelligence or predictive modeling may help us delineate when an interruption is warranted, for example, when a patient is at high clinical risk without intervention. Likewise, human factors research may help us understand the best way to time and execute an interruption to minimize the risk to clinical care or education. After all, the ideal system would not eliminate interruptions entirely but allow clinicians to know when someone should be interrupted and when they do not need to be interrupted.

Disclosures: The authors have no financial relationships relevant to this article to disclose.

\section{References}

1. Berner ES, Houston TK, Ray MN, et al. Improving ambulatory prescribing safety with a handheld decision support system: a randomized controlled trial. J Am Med Inform Assoc. 2006;13(2):171-179. doi: 10.1197/jamia.M1961.

2. Sintchenko V, Iredell JR, Gilbert GL, et al. Handheld computer-based decision support reduces patient length of stay and antibiotic prescribing in critical care. J Am Med Inform Assoc. 2005;12(4):398-402. doi: 10.1197/jamia. M1798.

3. Drolet BC. Text messaging and protected health information: what is permitted? JAMA. 2017;317(23):2369-2370. doi: 10.1001/jama.2017.5646.

4. Prochaska MT, Bird AN, Chadaga A, Arora VM. Resident use of text messaging for patient care: ease of use or breach of privacy? JMIR Med Inform. 2015;3(4):e37. doi: 10.2196/medinform.4797.

5. Samora JB, Blazar PE, Lifchez SD, et al. Mobile messaging communication in health care rules, regulations, penalties, and safety of provider use. JBJS Rev. 2018;6(3):e4. doi: 10.2106/JBJS.RVW.17.00070

6. Freundlich RE, Freundlich KL, Drolet BC. Pagers, smartphones, and HIPAA: finding the best solution for electronic communication of protected health information. J Med Syst. 2017;42(1):9. doi: 10.1007/s10916-017-0870-9.

7. Coiera E. Clinical communication-a new informatics paradigm. In Proceedings of the American. Medical Informatics Association Autumn Symposium. 1996; 17-21.

8. Feuerbacher RL, Funk KH, Spight DH, et al. Realistic distractions and interruptions that impair simulated surgical performance by novice surgeons. Arch Surg. 2012;147(11):1026-1030. doi: 10.1001/archsurg.2012.1480.

9. Agency for Healthcare Research and Quality-Patient Safety Network (AHRQ-PSNet). https://psnet.ahrq.gov/webmm/case/257/order-interrupted-by-text-multitasking-mishapCases \& Commentaries. Order Interrupted by Text: Multitasking Mishap. December 2011. Commentary by John Halamka, MD, MS

10. Westbrook JI, Raban MZ, Walter SR, et al. Task errors by emergency physicians are associated with interruptions, multitasking, fatigue and working memory capacity: a prospective, direct observation study [published online ahead of print January 9, 2018]. BMJ Qual Saf. doi: 10.1136/bmjqs-2017-007333. [Epub ahead of print].

11. Mendel A, Lott $A$, Lo $L$, et al. A matter of urgency: reducing clinical text message interruptions during educational sessions. J Hosp Med. 2018;13(9):616622. doi: 10.12788/jhm.2959. 